\title{
Unique Clinical and Histological Features of Placental Mesenchymal Dysplasia Complicated by Severe Preeclampsia in the Midtrimester
}

\author{
Yoshimitsu Kuwabara, MD, $\mathrm{PhD}^{1} \quad$ Mirei Yonezawa, MD ${ }^{1} \quad$ Yumene Kubota, MD \\ Tomoko Ichikawa, MD, $\mathrm{PhD}^{1}$ Ryuji Ohashi, MD, $\mathrm{PhD}^{2}$ Toshiyuki Takeshita, MD, $\mathrm{PhD}^{1}$

\footnotetext{
${ }^{1}$ Department of Obstetrics and Gynecology, Nippon Medical School

2 Department of Diagnostic Pathology, Nippon Medical School Musashi Kosugi Hospital, Kanagawa, Japan
} \\ Hospital, Tokyo Japan \\ Address for correspondence Yoshimitsu Kuwabara, MD, PhD, \\ Department of Obstetrics and Gynecology, Nippon Medical School, \\ 1-1-5 Sendagi, Bunkyo-ku, Tokyo 113-8602, Japan \\ (e-mail: kuwa@nms.ac.jp).
}

Am J Perinatol Rep 2020;10:e113-e117.

\begin{abstract}
Keywords

- placental mesenchymal dysplasia

- sFlt-1

- preeclampsia

- intrauterine growth restriction

Detailed clinical and pathological features of placental mesenchymal dysplasia (PMD) complicated by preeclampsia (PE) are unknown. A 39-year-old nulliparous woman was referred at 19 weeks gestation. Ultrasound examination identified a fetus with severe growth restriction (-2.8 SD) and a markedly thickened placenta with many cystic structures suggestive of PMD. At 21 weeks, the patient developed severe hypertension $(180 / 95 \mathrm{~mm} \mathrm{Hg})$ with marked proteinuria and an elevated serum soluble fms-like tyrosine-kinase- 1 (sFlt-1) level $(47,000 \mathrm{pg} / \mathrm{L})$. The pregnancy was terminated to protect maternal health. Placental weight was $450 \mathrm{~g}$ and no histopathological findings of either proliferation or dysplasia were observed in the trophoblast. Villous chromosome examination revealed a 46XX karyotype, consistent with the diagnosis of PMD. In addition to the pathological findings of vascular endothelial dysfunction characteristic of the placenta in PE cases, enhanced expression of sFlt- 1 in the syncytiotrophoblast of the enlarged villi was confirmed by immunohistochemistry as a novel finding in this condition. Monitoring of the serum sFlt-1 value is suggested to be a useful predictor of the pathological change associated with extremely early severe PE in PMD cases.
\end{abstract}

Placental mesenchymal dysplasia (PMD) is a rare anomaly characterized by an enlarged placenta with abnormal cystic villi similar to that seen in a hydatidiform mole. ${ }^{1}$ PMD should be considered when an apparently normal fetus with a cystic lesion of the placenta is observed by ultrasonography. However, differentiation from a complete mole with a co-twin is sometimes difficult. ${ }^{2}$ In spite of similar morphology to a hydatidiform mole, genetic and histological features are different and the fetal karyotype is usually normal. Unlike molar pregnancies, which are characterized by an absent or malformed fetus, pregnancy with PMD often extends to the third trimester without significant maternal morbidity. ${ }^{3}$
Therefore, termination of the pregnancy is usually inappropriate when PMD is suspected.

We, however, encountered a case of PMD in which a decision was made to terminate the pregnancy for the protection of the maternal health because of the development of severe preeclampsia (PE) in the second trimester of pregnancy. The case presented unique clinical features, including a marked elevation of serum soluble fms-like tyrosine kinase-1 (sFlt-1). To our knowledge, this is the first report demonstrating the histological findings of placental sFlt- 1 overexpression in such a condition. It provides a novel insight supporting the use of this biomarker to predict the extremely early development of severe PE in PMD. received

January 22, 2020

accepted

February 20, 2020
DOI https://doi.org/

10.1055/s-0040-1709186. ISSN 2157-6998.
Copyright $\odot 2020$ by Thieme Medical Publishers, Inc., 333 Seventh Avenue, New York, NY 10001, USA. Tel: +1(212) 760-0888.
License terms

(우(1) $\Theta \circledast$ 


\section{Case Report}

\section{Clinical Course}

A 39-year-old nulliparous woman (1G0P) was referred at 19 weeks of gestation because of a fetal morphological abnormality. On ultrasonography, the placenta was markedly thickened (- Fig. 1A) and multiple cystic structures without internal blood flow were observed (-Fig. 1B). The fetus was severely growth restricted ( $-2.8 \mathrm{SD}$ ) with an omphalocele. PMD was suspected, with a differential diagnosis of a hydatidiform mole with a coexisting fetus.

At 21 weeks and 2 days of gestation, the patient developed severe hypertension (180/95 mm Hg). Urine and blood examinations confirmed marked proteinuria (urinary protein/creatinine $=9.2)$, high uric acid $(0.47 \mathrm{mmol} / \mathrm{L})$, and an abnormally elevated sFlt level $(47,000 \mathrm{pg} / \mathrm{L}$, sFlt/placental growth factor ratio $=200$ ). Based on a diagnosis of severe extremely earlyonset $\mathrm{PE}$, termination of pregnancy was chosen to protect maternal health.

Stillbirth was induced by cervical dilatation using Laminaria followed by the intravaginal administration of prostaglandin E1, and the pregnancy was terminated at 21 weeks and 6 days of gestation. No external anomalies except for omphalocele were observed. Placental weight was $450 \mathrm{~g}$, the insertion site of the umbilical cord was marginal, and no vascular dilatation was observed on the fetal side of the placenta. Cystic structures similar to a molar pregnancy were observed in places on the maternal side of the placenta ( - Fig. 1C, D). Villous chromosome examination revealed a normal karyotype (46XX), consistent with the diagnosis of PMD. Maternal hypertension persisted for 2 weeks after delivery and oral administration of nifedipine was required during that period.

\section{Pathological Finding}

Microscopic findings of the placenta revealed focal enlarged stem villi with cistern and edematous loose myxoid stroma, surrounded by apparently normal villi with maturation consistent with the patient's gestational age ( $\mathbf{- F i g}$. 2A, B). Trophoblastic hyperplasia or inclusions, suggestive of molar pregnancy, were absent ( - Fig. 2C, D). Histological findings of decidual vessels revealed vasculopathy characterized by fibrinoid necrosis and infiltration of foamy macrophages in the vascular wall, either of which is characteristic of gestational hypertension and fetal growth restriction (-Fig. 3).

In the chorionic vessel lumen, the formation of fresh thrombus was present and marked luminal stenosis with organizing intimal sclerosis was noted in some of the chorionic vessels (-Fig. 4A, B).

Because of the abnormally high value of serum sFlt- 1 we investigated its expression in the placenta. Immunohistochemical staining of trophoblasts of PMD showed a high expression of sFlt-1 localized in the syncytiotrophoblast of
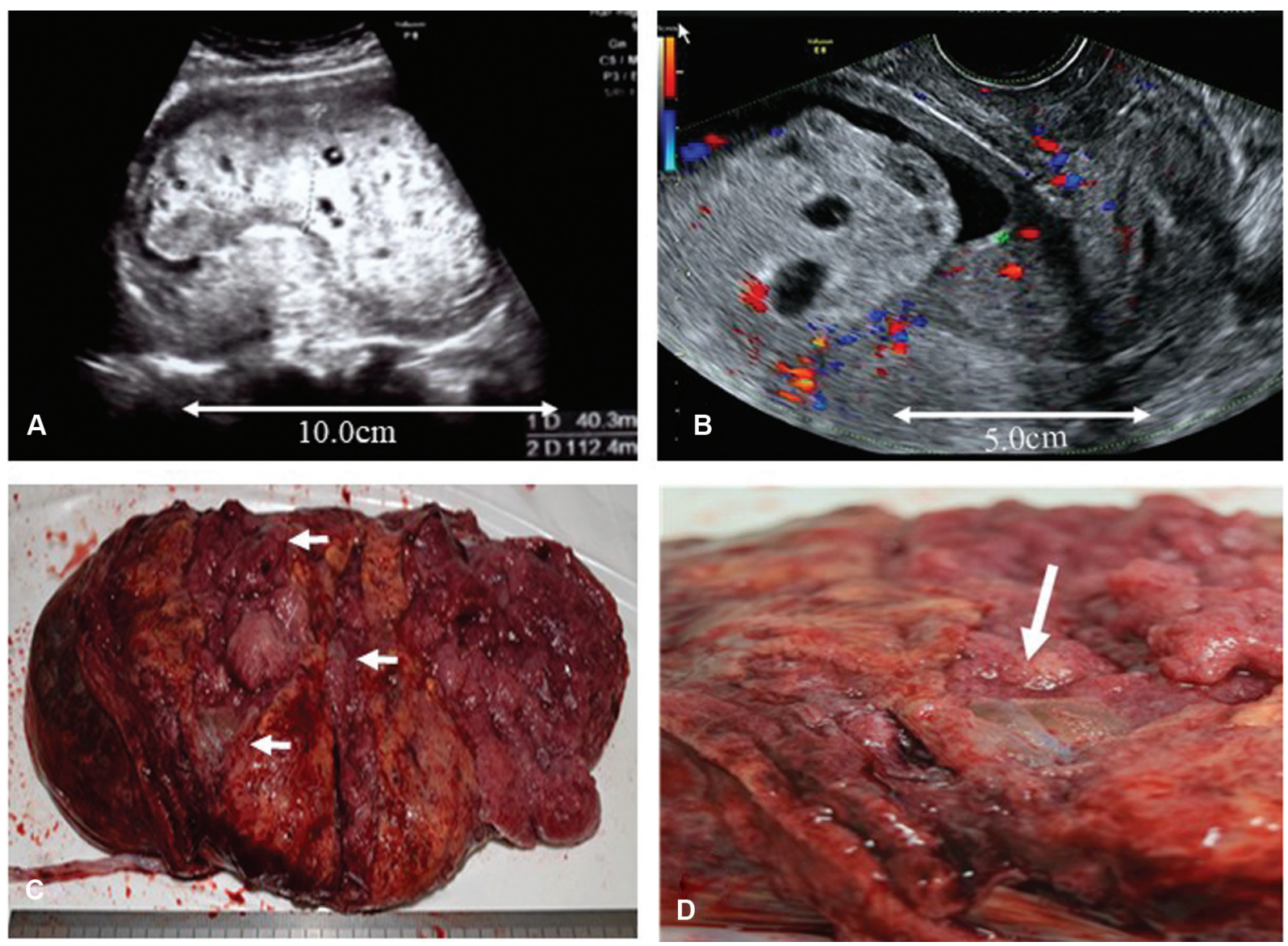

Fig. 1 Ultrasonographic findings of the placenta. (A) Markedly thickened placenta with multiple cystic structures. (B) No blood flow was observed in the cystic lesion. Macroscopic findings of the placenta. (C, D) Cystic structures similar to molar pregnancy observed in places on the maternal side of the placenta. 

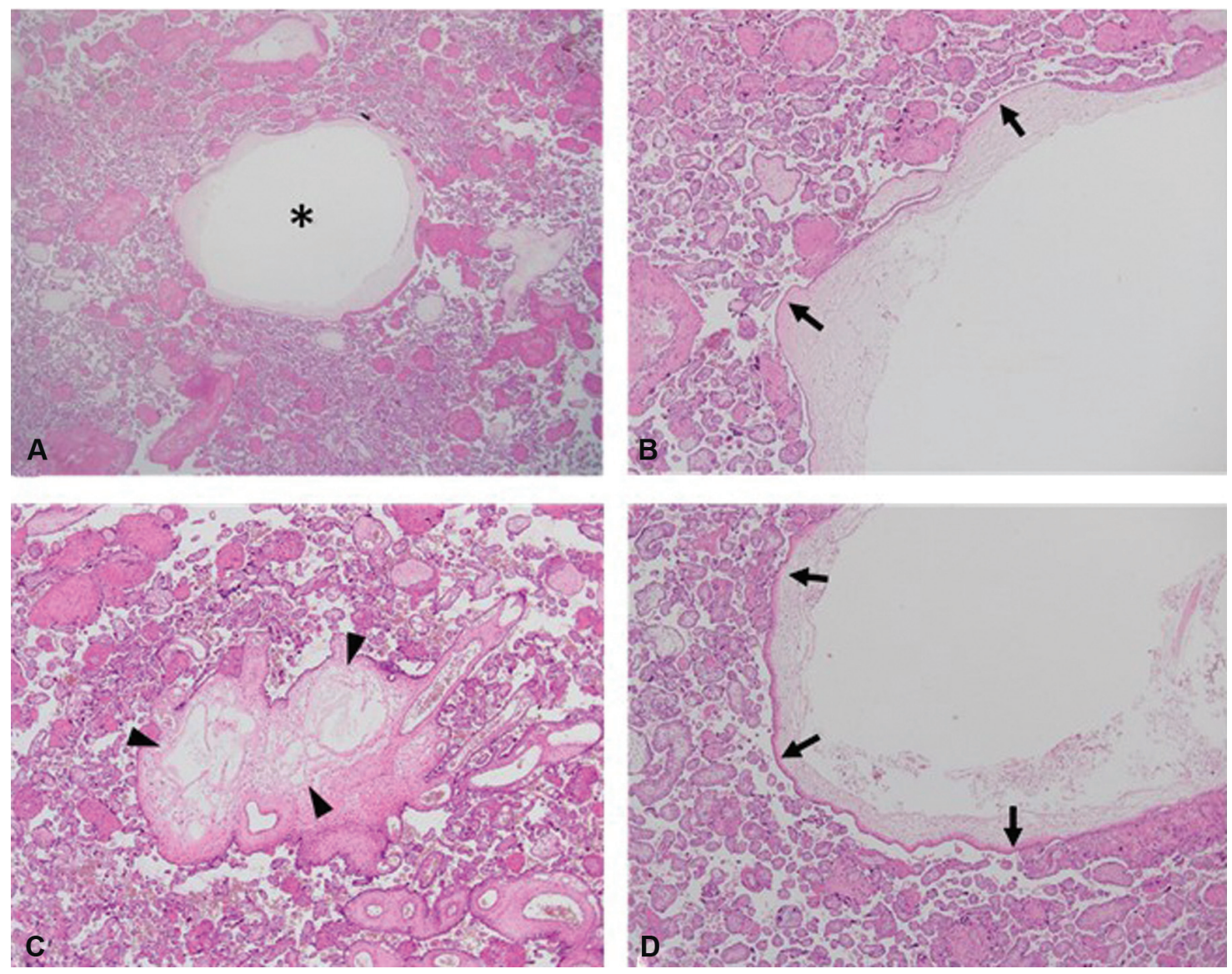

Fig. 2 Microscopic findings of the placenta. (A, B) Enlarged stem villi with cistern (asterisk) or edematous loose myxoid stroma (arrowheads) were focally present, surrounded by normal-appearing villi with gestational age-appropriate maturation. (C, D) Absence of trophoblastic hyperplasia or inclusions, suggestive of molar pregnancy (arrows) Original magnification A: $\times 12.5, B-D: \times 40$. A-D: Hematoxylin \& eosin stain.

the enlarged villi in PMD ( - Fig. 5A). However, its expression was weak in a control case terminated at 20 weeks of gestational age due to uterine rupture (-Fig. 5B).

\section{Discussion}

PMD is a unique placental anomaly first recognized by Takayama et al in $1986^{4}$ and later defined by Moscoso et al in $1991 .{ }^{1}$ Thereafter, a number of case reports have been published and the clinical features have been clarified. Characteristic findings are of a large placenta with multicystic anechoic lesions observed by ultrasonography and macroscopically recognized widely distributed edematous large villi, either of which is similar to that in a hydatidiform mole. ${ }^{1}$ Further, PMD is sometimes accompanied by a dilated vascular aneurysm on the fetal surface of the placenta, which was not observed in the present case. ${ }^{5}$ The histological finding is characterized by abnormally enlarged cystic stem villi in the absence of trophoblastic proliferation, which is the most obvious feature distinguishing it from hydatidiform mole, along with fetal karyotype. ${ }^{6}$ It is known that Beckwith-Wiedemann syndrome (BWS) is observed in about $20 \%$ of the cases; however, the difference in clinical characteristics from those with or without BWS has not been clarified. ${ }^{3}$ Because morphological information from the small fetus terminated in the midtrimester was inadequate and no diagnostic genetic testing for BWS was performed, it is unclear whether the present case had BWS.

In recent years, intrauterine growth restriction, late pregnancy loss, PE, and placental abruption have come to be regarded as a series of placenta-mediated pregnancy complications which develop in relation to placental hypoplasia and/or dysfunction. ${ }^{7}$ In a systematic review of PMD published in 2006 , about $5 \%$ of the cases were complicated by $\mathrm{PE}^{3}$ There are no reports in the literature of the detailed clinical course of patients with PMD complicated by PE, except for one case that in which the patient developed severe PE similar to the patient in the present case. ${ }^{8}$ Therefore, the clinical picture of this condition has not yet been elucidated.

Recently, the etiology of PE has been described as a vascular endothelial disorder originating from placental hypoplasia. The sFlt-1 produced from trophoblasts under hypoxic conditions is known to be a central mediator in the pathogenesis. ${ }^{9}$ Since serum sFlt- 1 values rise prior to the onset of PE, in recent years it has been used as a predictive marker. ${ }^{10}$ In addition to the pathological findings of vascular endothelial dysfunction characteristic of the placenta in PE 

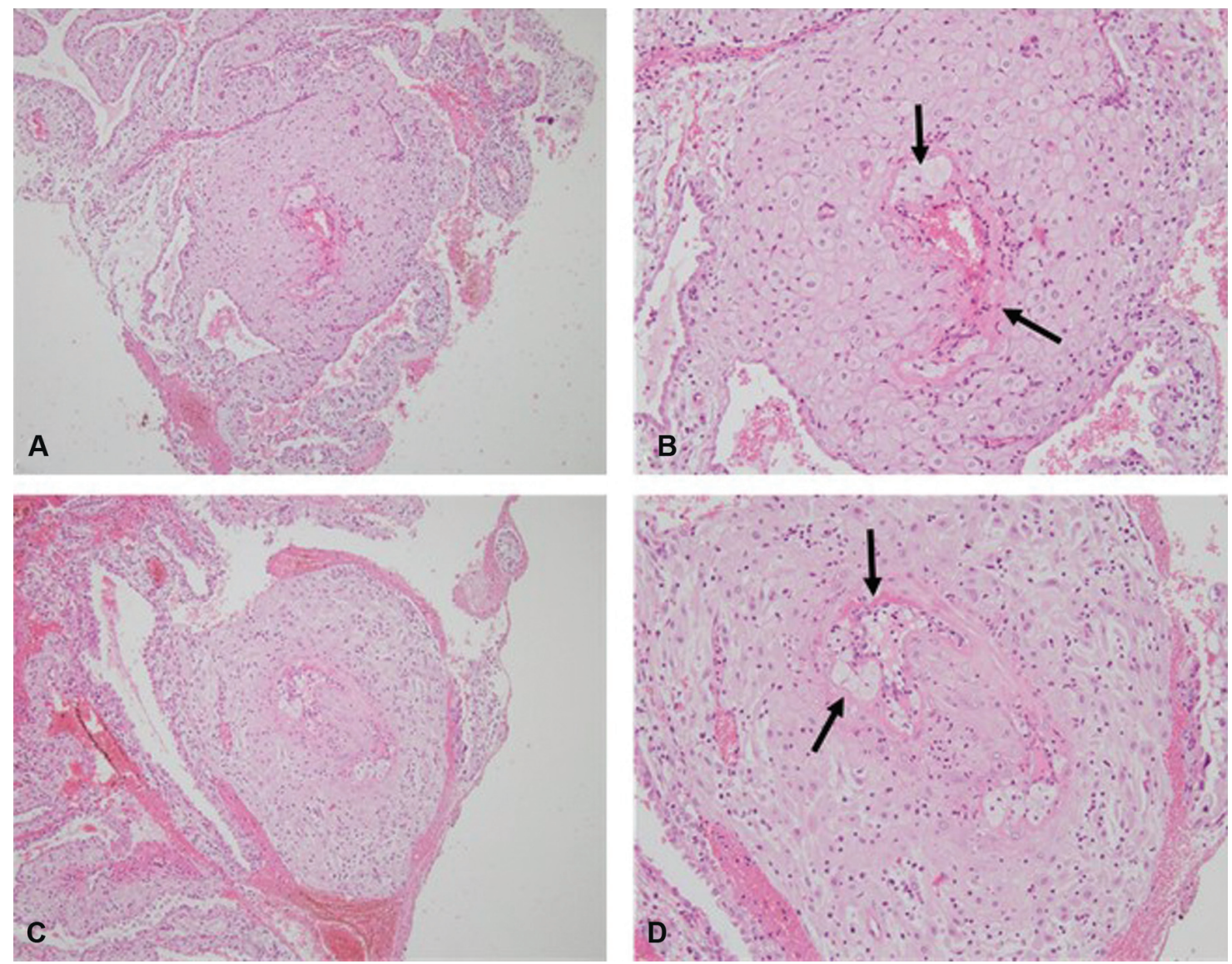

Fig. 3 Histological findings of decidual vessels. (A-D) Vasculopathy characterized by fibrinoid necrosis and infiltration of foamy macrophages (arrows) in the vascular wall Original magnification A, B: $\times 100, A, B:$ Hematoxylin \& eosin stain.
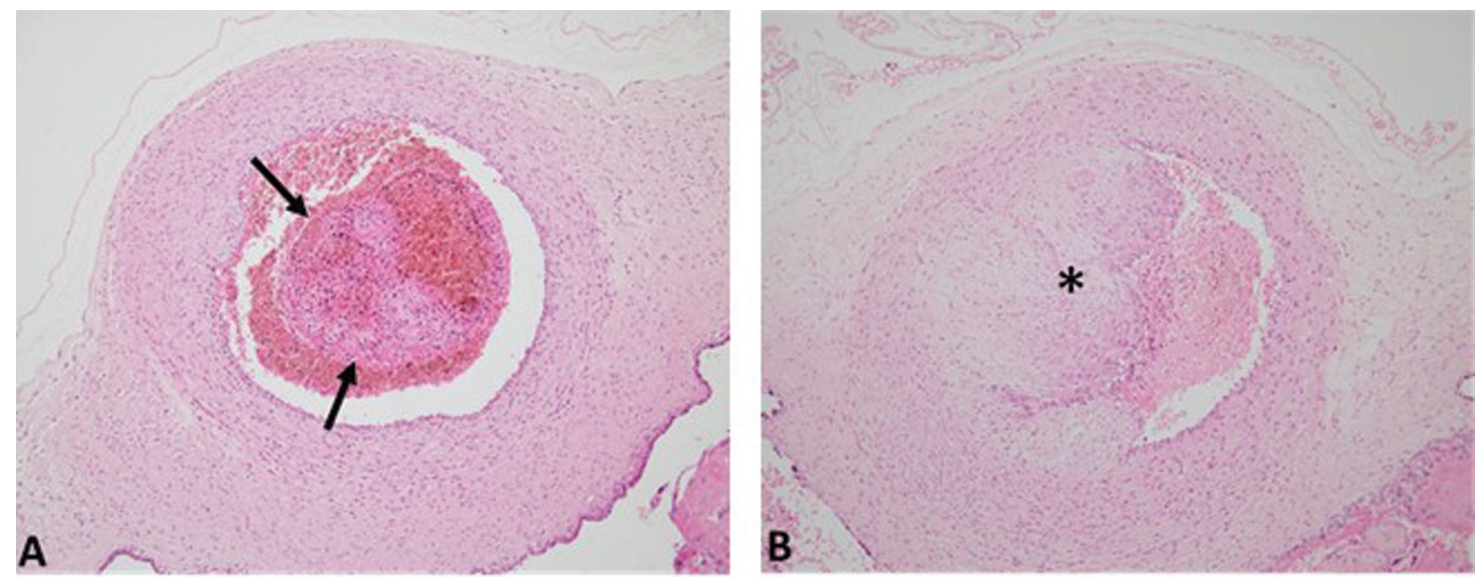

Fig. 4 (A) Formation of fresh thrombus in a chorionic vessel lumen (arrows). (B) Marked luminal stenosis with organizing intimal sclerosis in some of the chorionic vessels (asterisk) Original magnification A, B: $\times 100, C, D: \times 200$.

cases, the present case showed an overexpression of sFlt-1 localized in the trophoblast of enlarged villi. To our knowledge, this is the first report suggesting that the etiology of PE that develops in PMD cases is associated with the abnormal enhanced expression of sFlt- 1 in the syncytiotrophoblast of enlarged villi. When PMD is suspected, the clinician should keep in mind the possibility of development of extremely early severe PE and that monitoring the serum sFlt- 1 value may be useful in this regard.

\section{Conclusion}

In the case of placental findings similar to those of hydatidiform mole with a coexistent fetus, it is inappropriate to make an immediate decision to terminate the pregnancy, and it is important to evaluate it carefully and consider the possibility of PMD. More frequent antenatal assessments should take place if the pregnancy is to be continued, not only because of fetal growth restriction or late pregnancy loss, but also 

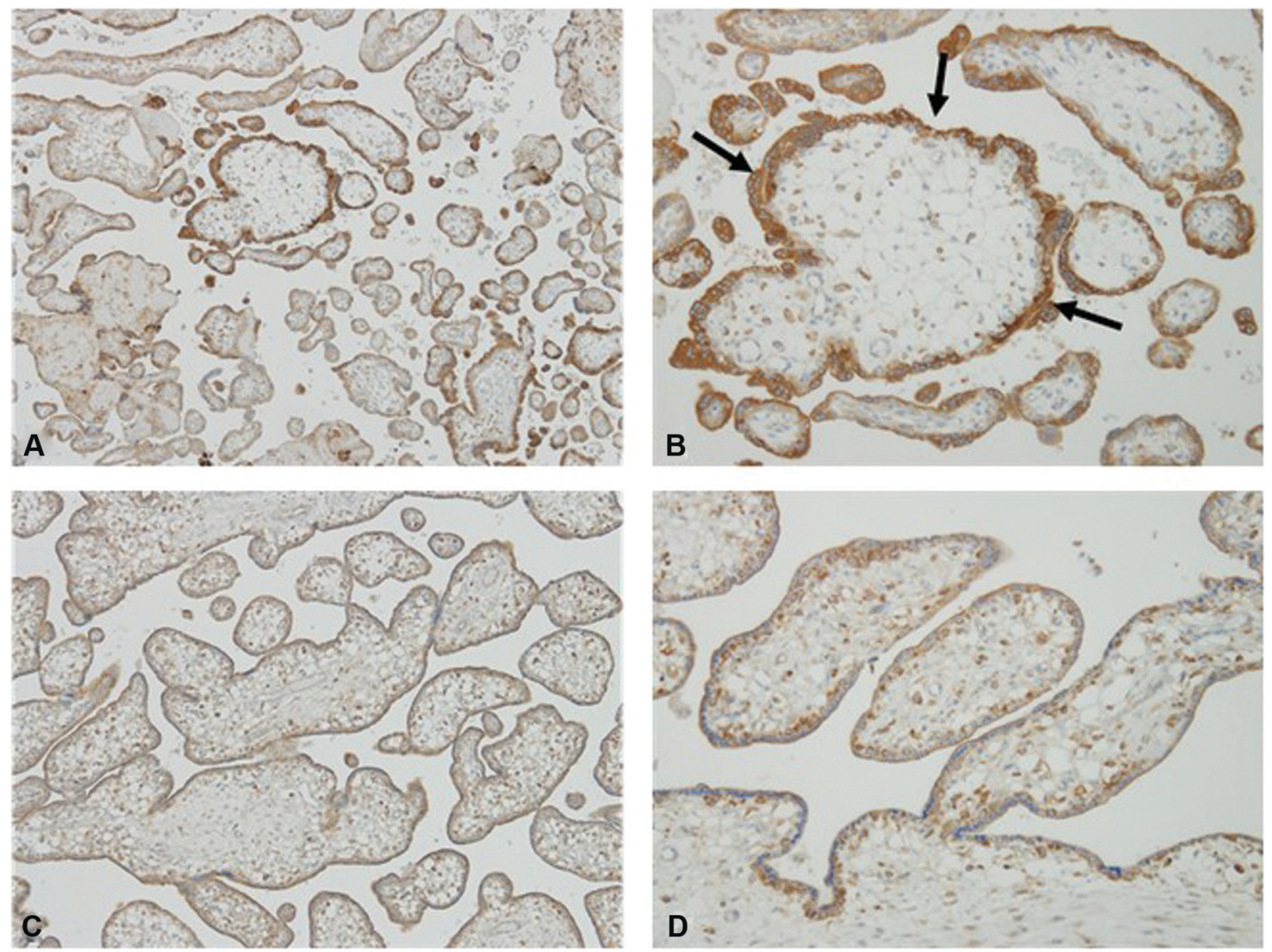

Fig. 5 Immunohistochemical staining of sFlt in trophoblasts of PMD (A, B) and control (C, D). Higher expression of sFlt was identified in the syncytiotrophoblast of enlarged villi in PMD (arrows), whereas its expression was weak in the control case, wherein the pregnancy was terminated at 20 weeks gestation due to uterine rupture. sFlt, soluble fms-like tyrosine-kinase-1; PMD, placental mesenchymal dysplasia.

because of the potential for very early onset of severe PE. The monitoring of serum sFlt-1 level is suggested to be beneficial for perinatal management in this condition.

\section{Conflict of Interest}

None.

\section{References}

1 Moscoso G, Jauniaux E, Hustin J. Placental vascular anomaly with diffuse mesenchymal stem villous hyperplasia. A new clinico-pathological entity? Pathol Res Pract 1991;187(23):324-328

2 Toru HS, Aytekin EÇ, Sanhal CY, et al. We can diagnose it if we consider it. Diagnostic pitfall for placenta: placental mesenchymal dysplasia. Turk Patoloji Derg 2018;34(01):100-103

3 Pham T, Steele J, Stayboldt C, Chan L, Benirschke K. Placental mesenchymal dysplasia is associated with high rates of intrauterine growth restriction and fetal demise: a report of 11 new cases and a review of the literature. Am J Clin Pathol 2006;126(01): $67-78$
4 Takayama M, Soma H, Yaguchi S, et al. Abnormally large placenta associated with Beckwith-Wiedemann syndrome. Gynecol Obstet Invest 1986;22(03):165-168

5 Kuwabara Y, Shima Y, Araki T, Shin S. Mesenchymal stem villous hyperplasia of the placenta and fetal growth restriction. Obstet Gynecol 2001;98(5 Pt 2):940-943

6 Surti U, Hill LM, Dunn J, Prosen T, Hoffner L. Twin pregnancy with a chimeric androgenetic and biparental placenta in one twin displaying placental mesenchymal dysplasia phenotype. Prenat Diagn 2005;25(11):1048-1056

7 Duffett L, Rodger M. LMWH to prevent placenta-mediated pregnancy complications: an update. Br J Haematol 2015;168(05):619-638

8 Na ED, Lee KJ, Kim M-J, Yi H-J, Kim J-Y. Placental mesenchymal dysplasia associated with severe preeclampsia: a case report. Korean J Obstet Gynecol 2011;54:459-463

9 Gu Y, Lewis DF, Wang Y. Placental productions and expressions of soluble endoglin, soluble fms-like tyrosine kinase receptor-1, and placental growth factor in normal and preeclamptic pregnancies. J Clin Endocrinol Metab 2008;93(01):260-266

10 Levine RJ, Lam C, Qian C, et al; CPEP Study Group. Soluble endoglin and other circulating antiangiogenic factors in preeclampsia. $\mathrm{N}$ Engl J Med 2006;355(10):992-1005 